\title{
ASYMMETRIC EXPRESSION PATTERNS OF BRAIN TRANSTHYRETIN IN NORMAL MICE AND A TRANSGENIC MOUSE MODEL OF ALZHEIMER'S DISEASE
}

\author{
K.-J. TSAI, ${ }^{a}$ C.-H. YANG, ${ }^{a, b}$ P.-C. LEE, ${ }^{a, c}$ W.-T. WANG, ${ }^{a}$ \\ M.-J. CHIU' AND C.-K. J. SHEN ${ }^{\mathrm{a}, \mathrm{b}, \mathrm{c} *}$ \\ a Institute of Molecular Biology, Academia Sinica, Nankang, Taipei 115, \\ Taiwan \\ ${ }^{b}$ Institute of Genome Sciences, National Yang-Ming University, Taipei, \\ Taiwan \\ 'Institute of Molecular Medicine, National Taiwan University, Taipei, \\ Taiwan \\ ${ }^{d}$ Department of Neurology, National Taiwan University Hospital, Tai- \\ pei, Taiwan
}

\begin{abstract}
Brain asymmetry is linked with several neurological diseases, and transthyretin (TTR) is a protein sequestering $\beta$-amyloid $(A \beta)$ and helping to prevent the Alzheimer's disease (AD). We show, by real time reverse transcriptionpolymerase chain reaction (RT-PCR), in situ hybridization and Western blotting, that TTR exhibits a pattern of adult male-specific, leftward distribution in the mouse brain. This asymmetry appeared to be mainly due to the asymmetric distribution of the choroid plexus cells in the ventricles. Unlike the normal mice, however, the hemispheric levels of TTR transcripts of 2- and 6-month-old Tg2576 mice, a transgenic $A D$ mouse model overexpressing $A \beta$, were symmetric in both sexes. Furthermore, at the age of 10 months when the pathological AD-like features had developed, the level of TTR transcripts in the left hemisphere of the male Tg2576 became significantly lower than the right one. This lowering of TTR transcript is accompanied with a higher $A \beta$ level in the left hemisphere of the 10-month Tg2576 males. Finally, for both genders, the TTR transcript levels in the two hemispheres of aged Tg2576 mice were lower than either the adult Tg2576 or the aged nontransgenic controls. Based on the above, we suggest scenarios to correlate the changes in the levels and hemispheric patterns of TTR expression to the pathogenesis of AD. () 2009 IBRO. Published by Elsevier Ltd. All rights reserved.
\end{abstract}

Key words: Alzheimer's disease, asymmetry, gene expression, mouse brain, transthyretin.

Transthyretin, or TTR, is the most abundant protein expressed in the choroids plexus of the brain (Sousa et al., 2007) in mammals, birds, and reptiles (Schreiber, 2002). This evolutionary conservation suggests important role(s)

${ }^{*}$ Correspondence to: C.-K. J. Shen, Institute of Molecular Biology, Academia Sinica, Nankang, Taipei 115, Taiwan. Tel: +886-2-27824188; fax: +886-2-2788-4177.

E-mail address: ckshen@imb.sinica.edu.tw (C.-K. J. Shen).

Abbreviations: AD, Alzheimer's disease; APP, amyloid precursor protein; $A \beta, \beta$-amyloid; BBB, blood-brain barrier; GAPDH, glyceraldehyde-3-phosphate dehydrogenase; $\mathrm{HE}$, hematoxylin and eosin; IMB, Institute of Molecular Biology; RT-PCR, reverse transcription-polymerase chain reaction; TTR, transthyretin.

0306-4522/09 @ 2009 IBRO. Published by Elsevier Ltd. All rights reserved. doi:10.1016/j.neuroscience.2008.12.045 played by TTR in the mammalian brain. TTR is one of the principal carriers in the plasma for the thyroid hormones (Palha et al., 1994), which are essential for the development and function of the mammalian brain (Schreiber, 2002). TTR expressed in the choroid plexus may carry thyroid hormone $\mathrm{T}_{4}$ into the CNS through the blood-brain barrier (BBB) (Chanoine et al., 1992). Importantly, TTR protein has been shown to bind and sequester $A \beta$ to prevent its aggregation (Schwarzman et al., 1994; Link, 1995) thus playing a neuroprotective role in $A D$ (Stein et al., 2004; Buxbaum et al., 2008). On the other hand, TTR gene expression could be induced in response to overproduction of $A \beta$ (Stein and Johnson, 2002). This seems to be in contrast to the observations that the concentration of TTR is lower in most, if not all, AD patients than in agematched controls (Serot et al., 1997; Chodobski and Szmydynger-Chodobska, 2001).

Accumulating evidence has indicated that human brain is indeed asymmetrical in anatomical, functional, as well as neurochemical aspects (Galaburda et al., 1978; Geroldi et al., 2000b, Hugdahl, 2000, Toga and Thompson, 2003). Differences between the left and right hemispheres have been implicated in many aspects of the brain function and dysfunction, including the developmental disorders of schizophrenia, epilepsy, dyslexia and AD (Barta et al., 1997; Geroldi et al., 2000a). It has also been hypothesized that failure to develop proper asymmetry is an important factor of the pathology underlying some forms of neurological diseases (Geschwind and Miller, 2001; Toga and Thompson, 2003). In particular in patients with AD, for example, loss of asymmetry in the hippocampus has been observed (Geroldi et al., 2000b). In addition, the atrophy of the right parahippocampal gyrus is deteriorated in AD patients (Pantel et al., 2003). In those AD patients with delusions, a reversed pattern of temporal and frontal horn asymmetries were found (Geroldi et al., 2000a).

The presence of brain asymmetries in mammals other than human (Tabibnia et al., 1999; Sun and Walsh, 2006) opens the possibility for molecular and cellular research on the structure-function of brain asymmetry by using animals such as mouse as the model system (Kawakami et al., 2003; Sun et al., 2005). As shown below, we have identified TTR as a gene exhibiting an asymmetric expression pattern of its transcripts in the male mouse brains, but not in the females. Interestingly, this pattern of asymmetric TTR expression was altered in aged mice as well as in a transgenic mouse model of $A D$. 


\section{EXPERIMENTAL PROCEDURES}

\begin{abstract}
Animals
Eight-week-old C57BL/6JNarl mice were purchased from the National Laboratorial Animal Center (Taipei, Taiwan), while transgenic Tg2576 mice and non-transgenic littermates were purchased from Taconic (Germantown, NY, USA). The mice were bred at the Animal Facility of the Institute of Molecular Biology (IMB), Academia Sinica, Taiwan. They were housed in a room maintained on a 12-h light/dark cycle (light on at 7.00 AM) with continuous supply of food and water. The use of animals in this study was approved by the Institutional Animal Care and Use Committee in IMB. Experimental procedures for handling the mice also followed the guidelines of IMB. The authors attest that all efforts were made to minimize the number of animals used and their suffering.
\end{abstract}

\section{Tissue preparation}

Each mouse was perfused with $20 \mathrm{ml} 0.1 \mathrm{M}$ PBS and $20 \mathrm{ml} 4 \%$ paraformaldehyde/0.1 M PBS. The brain was taken out immediately and incubated in $4 \%$ paraformaldehyde/0.1 M PBS for an hour, and then soaked in $20 \%$ sucrose $/ 4 \%$ paraformaldehyde for at least 1 day.

\section{RNA preparation}

The left and right cerebral hemispheres were dissected from normal C57BL/6JNarl mice or the Tg2576 AD mice at different ages. They were lysed in TRIzol REAGENT (Invitrogen, Carlsbad, CA, USA) following the manufacturer's protocol. After homogenization, the homogenates were extracted with chloroform and isopropanol, and precipitated in ethanol. The final RNA solutions were further cleaned up using the RNeasy kit (QIAGEN, Valencia, CA, USA) as well as with a DNase treatment step to remove the contaminated DNA. The concentrations of the RNAs were determined by Ultraspec 3000 spectrophotometer (Amersham Pharmacia Biotech, Piscataway, NJ, USA), and their qualities were validated by measuring the ratios of the 28 S rRNA/18 S rRNA in an Agilent (2100) BioAnalyzer (Agilent Technologies, Santa Clara, CA, USA).

\section{DNA microarray hybridization}

The RNAs of the left and right hemispheres, respectively, of two adult male mice were isolated. Microarray hybridization experiments were then carried out, each using $20 \mu \mathrm{g}$ of the RNAs from the left and right hemispheres, respectively. The probe labeling and hybridization were carried out at the DNA MicroArray Facility of the University of California, Irvine. In brief, the RNA samples were reverse transcribed into double-stranded cDNA using the Superscript Choice kit (Invitrogen, Carlsbad, CA, USA) containing a T7-DT 24 primer that would incorporate a T7 RNA polymerase promoter during reverse transcription. The CRNAs were biotinlabeled during in vitro transcription (Enzo Biochem, New York, NY, USA), and used as the probes for hybridization with the MGU74Av2 arrays containing 12,473 oligo probe sets (Affymetrix, Santa Clara, CA, USA). After hybridization, the gene chips were washed and stained with streptavidin-phycoerythrin on a fluidics station. Finally, the probed arrays were scanned at $3 \mu \mathrm{m}$ resolutions in the Genechip System Confocal Scanner (Affymetrix, Santa Clara, CA, USA). Affymetrix Microarray Suite 4.1 was used to scan and analyze the relative abundance of each gene based on the average differences of the intensities (Lipshutz et al., 1999). The candidate genes were selected based on two criteria: First, the signals must be called as "present" in as least one set of the samples; second, the change of the expression level must be determined as "increase" with a signal log ratio $\left(\log _{2} \mathrm{~L} / \mathrm{R}\right)$ above 0.1 , or as "decrease" with a signal log ratio below -0.1 . Finally, the definitions of "increase," "decrease," or "no change of expres- sion" for the individual genes were based on the three intergroup comparisons between the duplicate hybridization experiments.

\section{RT-PCR}

Both real time RT-PCR and semi-quantitative RT-PCR were used to compare the mRNA levels. For each reaction of first-strand cDNA synthesis, $0.5 \mu \mathrm{g}$ of the total RNA were mixed with $500 \mathrm{ng}$ of oligo (dT) $)_{18}$ and $0.5 \mathrm{mM}$ dNTP mixture. The mixture was heated to $65^{\circ} \mathrm{C}$ for $5 \mathrm{~min}$ and quickly chilled on ice before addition of the $5 \times$ first-strand buffer, $10 \mathrm{mM}$ DTT, and $40 \mathrm{U}$ of ribonuclease inhibitor (Promega, Madison, WI, USA) in a total volume of $19 \mu \mathrm{l}$. The mixture was incubated at $42{ }^{\circ} \mathrm{C}$ for $2 \mathrm{~min}$, added with $1 \mu \mathrm{l}$ of SuperScript II (Invitrogen), and then incubated for another $70 \mathrm{~min}$ at $42{ }^{\circ} \mathrm{C}$. Ten-fold diluted cDNAs were used as the templates for PCR.

The real time PCR was then performed with $25 \mathrm{ng}$ of CDNA in an Applied Biosystems (AB, Foster City, CA, USA) 7500 Sequence Detection System using the AB Taqman Gene Expression Assays for TTR (Mm00443267-m1, the amplicon length of which was 62 bp and its boundaries were from exon 3 to exon 4) and primers-limited glyceraldehyde-3-phosphate dehydrogenase (GAPDH) (Mm99999915-g1), respectively. No significant differences in GAPDH expression were detected among the different groups of samples. The threshold amplification cycle number (ct) data from multiple plates were combined using AB Relative Quantitation software (SDS1.4) and the $\Delta \Delta \mathrm{Ct}$ method with GAPDH as the endogenous control. All data were expressed as the mean fold-of-change $\pm S E$.

For semi-quantitative PCR, the forward and reverse primers used for TTR were 5' -CCT GCT CAG CCC ATA CTC CTA-3' and 5'-AAC GGT TGG TCC ACT CTG CT-3', respectively, both of which were derived from exon 4. The primers for GAPDH were 5'-GAC CCC TTC ATT GAC CTC AAC- $3^{\prime}$ and 5'-TCT TAC TCC TTG GAG GCC ATG-3'. The PCR reactions were initiated by denaturation at $94{ }^{\circ} \mathrm{C}$ for $3 \mathrm{~min}$, followed by 32 cycles at $94{ }^{\circ} \mathrm{C}$ for $30 \mathrm{~s}, 60{ }^{\circ} \mathrm{C}$ for $30 \mathrm{~s}$, and finally at $72{ }^{\circ} \mathrm{C}$ for $30 \mathrm{~s}$. The PCR products were allowed to be extended at $72{ }^{\circ} \mathrm{C}$ for $10 \mathrm{~min}$, and the products were resolved on $1.5 \%$ agarose gels. The images were captured and densitometry was performed by using the Alpha Imager 2200 (Alpha Innotech Corporation, San Leandro, CA, USA). The TTR levels were then compared after calibration against the corresponding GAPDH levels.

\section{In situ hybridization}

In situ hybridization was performed according to the procedures described previously (Tsai et al., 2002) with slight modifications. Brain coronal sections with the width of $20 \mu \mathrm{m}$ were taken from the forebrain to the hind-brain serially, covering the hippocampus and the ventricles (central and lateral) of the cerebrum. The antisense probe complementary to nucleotide 307-351 of the TTR cDNA (5'-CAT GGA ACG GGG AAA TGC CAA GTG TCT TCC AGT ACG ATT TGG TGT-3') and the sense probe were $3^{\prime}$ end-labeled with $\alpha\left[{ }^{35} \mathrm{~S}\right] \mathrm{dATP}$ and hybridized, respectively, at $42{ }^{\circ} \mathrm{C}$ for $24 \mathrm{~h}$ with the silane-coating slides (Muto Pure Chemicals Co., New York, NY, USA) containing at least 50 brain sections. After extensive washing, the slides were dehydrated with ethanol and exposed to Hyperfilm MP (Amersham Pharmacia Biotech, Piscataway, NJ, USA) for 10 days. The signals from the in situ hybridization were quantified by measuring the optic densities of the relevant fields with the use of the National Institutes of Health Image program.

\section{Western blotting}

The left and right hemispheres of the male and female mice at the age of 2 months were homogenized in RIPA lysis buffer [Tris- $\mathrm{HCl}$ $50 \mathrm{mM}, \mathrm{NaCl} 150 \mathrm{mM}$, Igepal CA-630 1\%, EDTA (pH 8.0) 2 mM, 


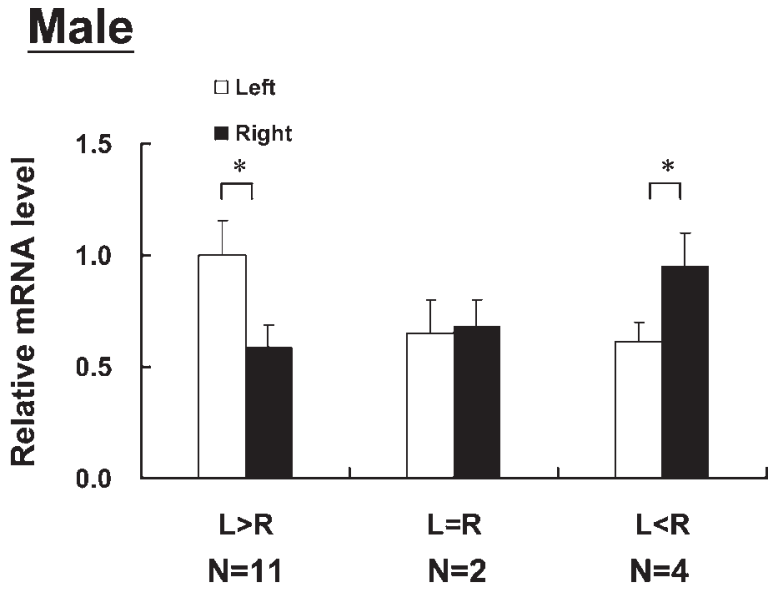

Female

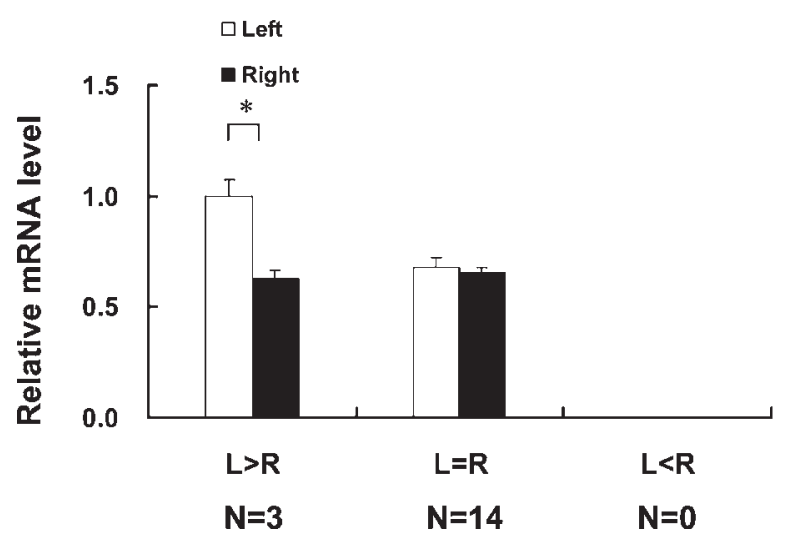

Fig. 1. Real time RT-PCR analysis of the cerebral hemispheres of adult mice. The distribution of TTR transcripts in the left $(\mathrm{L})$ and right (R) hemispheres of 17 each of adult male mice and adult female mice was analyzed by real time RT-PCR. Gene expression was normalized to GAPDH as an endogenous control. Note that a significantly higher percentage of male mice have a leftward, asymmetric pattern of TTR expression.

Na3VO4 $1 \mathrm{mM}$, pepstain A $20 \mu \mathrm{g} / \mathrm{ml}$, leupeptin $20 \mu \mathrm{g} / \mathrm{ml}$, aprotinin $20 \mu \mathrm{g} / \mathrm{ml}$, PMSF $1 \mathrm{mM}$, NaF $50 \mathrm{mM}$ ]. Equal amounts of the total protein extracts were then analyzed by Western blotting with use of an anti-TTR antibody from Abcam. The relative density of each band was normalized against that of the tubulin.

\section{Hematoxylin and eosin (HE) staining}

To locate the choroid plexus cells (Stein and Johnson, 2002), the brain sections were stained with hematoxylin for 2 min and then eosin for $30 \mathrm{~s}$. After washing, the slides were dehydrated and sealed with Entellan (Merck, Whitehouse Station, NJ, USA) before examination under the light microscope. Images of the choroid plexus from multiple sections in each mouse were captured under identical light levels and stored for later cell density measurement. Quantitation of the cell densities were done with the AlphaEaseFC software (Alpha Innotech Corporation, San Leandro, CA, USA).

\section{Quantification of the $A \boldsymbol{\beta}$ level}

The level of $A \beta$ was quantified following the procedures of Tsai et al. (2007). The tissue samples were homogenized in 10 wetweight volumes of PBS containing a cocktail of protease inhibitors $(20 \mu \mathrm{g} / \mathrm{ml}$ each of pepstatin A, aprotinin, phosphoramidon and leupeptin, $0.5 \mathrm{mM}$ PMSF, and $1 \mathrm{mM}$ EGTA). The samples were then sonicated briefly $(10 \mathrm{~W}, 2 \times 5 \mathrm{~s})$ and centrifuged at $100,000 \times \mathrm{g}$ for $20 \mathrm{~min}$ at $4{ }^{\circ} \mathrm{C}$. The soluble fraction (supernatant) was subjected to $A \beta$ ELISA assay (Sigma-Aldrich, St. Louis, MO, USA). To quantitate the insoluble $A \beta / A \beta$ aggregates, the SDS-insoluble pellet was sonicated and dissolved in $70 \%$ formic acid (FA). The extract was then neutralized with $0.25 \mathrm{M}$ Tris, pH 8.0, containing $30 \%$ acetonitrile and $5 \mathrm{M} \mathrm{NaOH}$ before loading onto an ELISA plate. The protein-containing samples were subjected to assay by the $A \beta$ ELISA kit (sigma BE0200) and the signals at $450 \mathrm{~nm}$ were detected using a multiwell plate reader.

\section{RESULTS}

\section{DNA microarray analysis of the symmetry of brain gene expression}

To search for asymmetrically expressed genes in the cerebral hemispheres of adult mice, we have carried out gene profiling of the mouse hemispheres with use of the Affymatrix microarray system (see Experimental Procedures for details). Preliminary, duplicated hybridization experiments have suggested that TTR is an "asymmetrically expressed" gene in the adult mouse brain (Tsai, unpublished observations). Because of its implication in the sequestering of $A \beta$ aggregates and the prevention of $A D$ (see Discussion later), the asymmetric expression of TTR in the mouse brain was subjected to further analysis by real time RT-PCR, in situ hybridization, and Western blotting.

\section{Individual randomization and gender specificity of the asymmetric expression of TTR transcripts in the mouse brain}

To determine whether the asymmetry of the TTR expression was a general phenomenon in individual mice and whether it might be gender specific, we first used real time RT-PCR to examine RNAs isolated from the left and right cerebral hemispheres of 17 adult male mice and 17 adult female mice, respectively. As shown in Fig. 1 and Table 1, the distributions of TTR transcripts in the hemispheres exhibited a pattern of leftward asymmetry in the adult males, with the levels of TTR RNA higher in the left cerebral hemispheres of 11 of the mice analyzed. In contrast,

Table 1. Summary of TTR expression profiles in the cerebral hemispheres of adult mouse brain

\begin{tabular}{|c|c|c|c|c|c|c|}
\hline & \multicolumn{3}{|c|}{ Male $(N=30)$} & \multicolumn{3}{|c|}{ Female $(N=24)$} \\
\hline & $L>R$ & $L=R$ & $\mathrm{~L}<\mathrm{R}$ & $\mathrm{L}>\mathrm{R}$ & $\mathrm{L}=\mathrm{R}$ & $\mathrm{L}<\mathrm{R}$ \\
\hline RT-PCR & 11 & 2 & 4 & 3 & 14 & 0 \\
\hline In situ hybridization & 7 & 3 & 3 & 3 & 4 & 0 \\
\hline Total & 18 & 5 & 7 & 6 & 18 & 0 \\
\hline Percentage & $60 \%$ & $16.7 \%$ & $23.3 \%$ & $25 \%$ & $75 \%$ & 0 \\
\hline
\end{tabular}

The expression of TTR in the cerebral hemispheres of 30 adult male mice and 24 adult female mice was analyzed by either RT-PCR or in situ hybridization. The patterns of asymmetry/symmetry are categorized according to whether the level of TTR transcripts is higher in the left hemisphere $(L>R)$, higher in the right hemisphere $(L<R)$, or equal on both sides of the cerebrum $(L=R)$. The percentages of the individual categories are calculated for the male and female mice, respectively. 
the majority (14/17) of the female mice exhibited a symmetric distribution pattern of the TTR transcripts (Fig. 1, Table 1). Semi-quantitative RT-PCR analysis gave similar results (data not shown).

In the real time RT-PCR experiments described above, the levels of the TTR transcripts between the two hemispheres usually differed by 1.5 -fold or more whenever an asymmetry was found (Fig. 1A). This is most likely due to the differential amounts of TTR transcripts in the choroid plexus of the two bilateral ventricles, the region within which TTR is synthesized in the brain. It is also the brain region with the most abundant levels of TTR RNA (Stauder et al., 1986; Lein et al., 2007) as well the TTR protein (Stauder et al., 1986). To see if this was indeed the case, in situ hybridization was used to delineate the distributions of TTR transcripts in individual adult mouse brains. A series of sections at $20 \mu \mathrm{m}$ intervals from the forebrain to the hind-brain of the individual brains was hybridized with TTR-specific antisense probe. An example is shown in Fig. $2 \mathrm{~A}$ for an adult male mouse. We found that signals could be detected in the ventricles at the locations of the choroid plexus. Furthermore, the relative levels of the TTR tran-
A

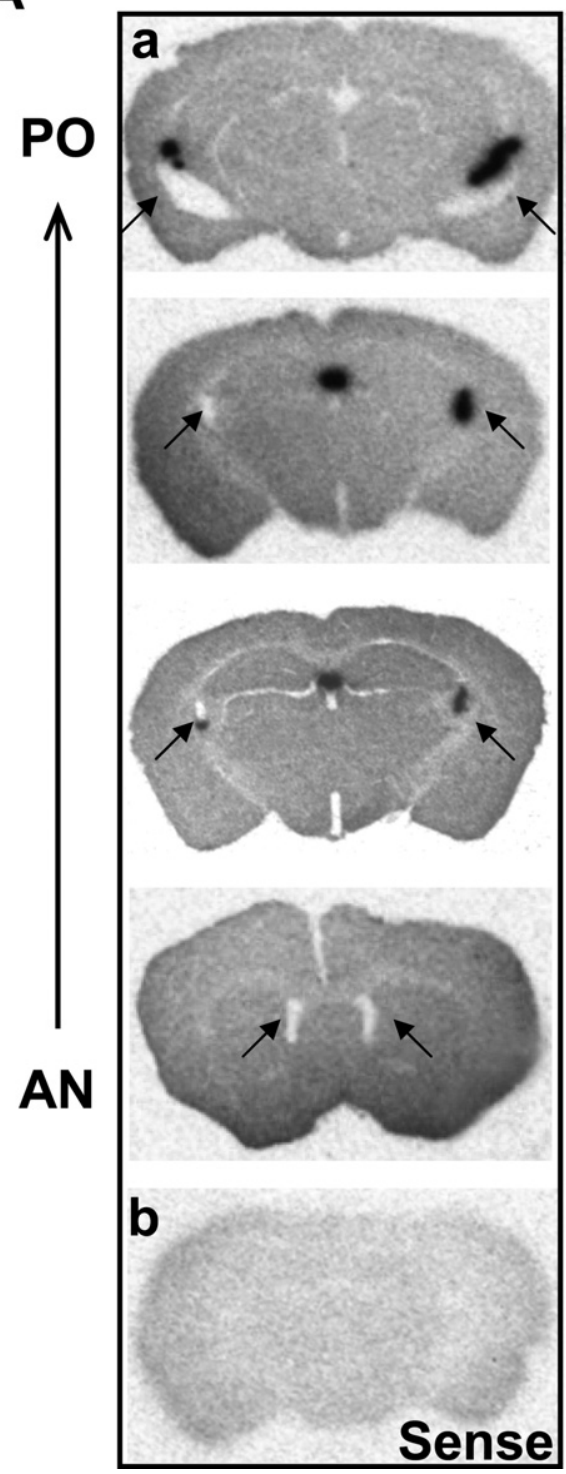

B

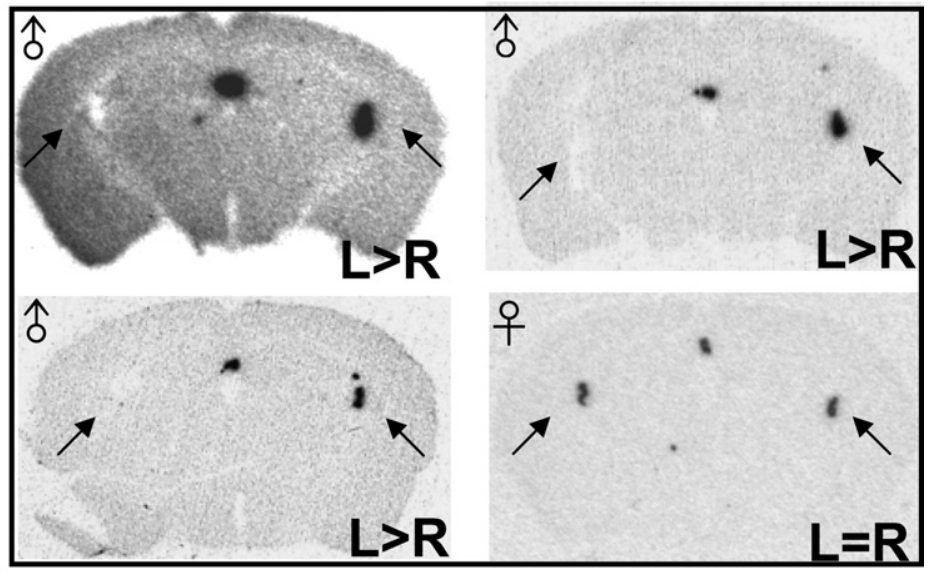

C

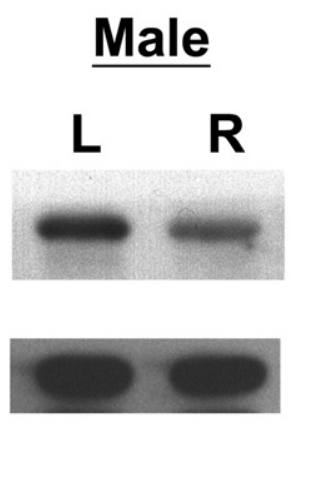

Female

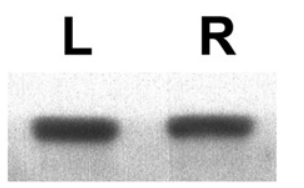

TTR

Tubulin

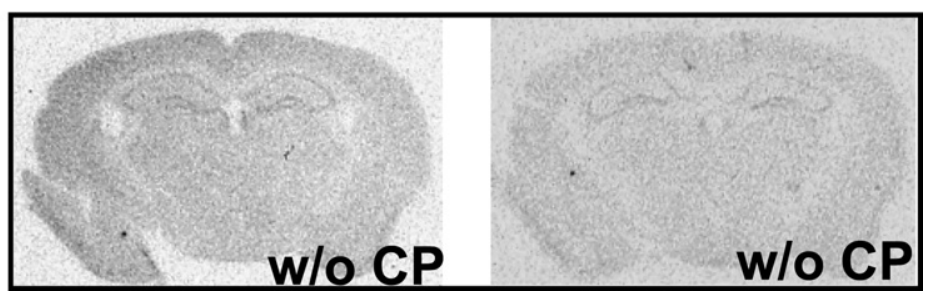

Fig. 2. Distribution pattern of TTR transcripts in the brain of an adult male mouse. In situ hybridization was used to examine the distribution pattern of TTR transcripts in the cerebrum of a male mouse of the age 2 months. (A) The autoradiographs of four coronal sections (AN $\rightarrow P O$, anterior to posterior). Note that there is also a central ventricle, but only the lateral ventricles are indicated by the arrows. (A) The image of a coronal section hybridized with the sense probe. (B) Distribution patterns of mouse brain TTR transcripts in three males and one female. Representative autoradiographs from in situ hybridization experiments of three adult male mice (upper and lower-left panels) and one adult female mouse (lower-right panel) are exemplified. Note the asymmetric distribution of the TTR transcripts in the three male brains, in contrast to the symmetric pattern in the female. (C) A representative Western blotting analysis of the mouse brain extracts of one adult male and one adult female. Note the asymmetric pattern of TTR protein distribution in the male brain. (D) Long exposure patterns of TTR RNA distribution in two consecutive coronal sections with the choroid plexus removed prior to the in situ hybridization (w/o CP). Note the weak TTR signals in the hippocampus. 
scripts in the two ventricles were usually consistent among the different sections of the same brain, as exemplified in Fig. 2A for an adult male that had a higher amount of TTR transcripts in the left ventricle. Representative sections of three more adult male brains and one adult female brain are shown in Fig. 2B. The leftward asymmetry of TTR expression in the adult male mouse brains was also observed on the protein level, as exemplified in Fig. 2C.

Interestingly, while the majority of brain TTR RNAs were localized in the choroid plexus, very low level of TTR transcripts could be detected in the hippocampus, as exemplified for an adult male brain with the choroid plexus removed before the in situ hybridization analysis (Fig. 2D). This is in agreement with the studying by Stein and Johnson (2002) and Lein et al. (2007) but not that by Sousa et al. (2007). Furthermore, no asymmetry in the distribution of the TTR transcripts in brain regions other than the choroid plexus could be observed (Fig. 2D; data not shown).

In the combined analyses by real time RT-PCR and in situ hybridization, as summarized in Table 1, we have found that the adult male mice exhibited an individually randomized asymmetry of TTR expression, with 18 (60\%) mice of the leftward asymmetry, 7 (23.3\%) rightward, and $5(16.7 \%)$ of them symmetric. On the other hand, TTR expression in the brains of a majority $(75 \%)$ of the female mice analyzed was symmetrical, and $25 \%$ were of the leftward expression pattern. The data of Fig. 1 and Table 1 were analyzed by the $\chi^{2}$-test and the results $(P<0.05)$ indicated the statistical significance of the differences between the two groups.

\section{Basis for the asymmetric expression of TTR}

We have attempted to find out the basis underlying the asymmetric TTR expression in the mouse brain. Choroid plexus, the major synthesis site for TTR, consists of the continuous epithelial cells that project into the ventricles and release TTR into the cerebrospinal fluid. The irregular configuration of the hanged epithelial cells led us to wonder whether the asymmetrical signals of TTR in the ventricle areas, as detected by in situ hybridization, reflected the differences in the distributions of these cells. To check this, we stained the same slides used for in situ hybridization with $\mathrm{HE}$, which could delineate the cell histology of the brain sections. Interestingly, for the adult mice analyzed, the relative densities of the epithelial cells in the choroid plexus of the left and right ventricles, respectively, appeared to be coincident with the relative levels of the in situ hybridization signals, as exemplified in Fig. 3 for a brain with leftward expression of TTR. The data indicated that the asymmetric expression of TTR in the brain was mainly due to the asymmetric distribution of the choroid plexus cells in the left and right ventricles of the individual mouse brains.

\section{Expression patterns of TTR in the brains of aged C57BL/6JNarl mice and Tg2576 mice}

Results from the above experiments demonstrated that TTR expression was asymmetrical in the cerebral hemispheres, especially in the male mice. Since one of the
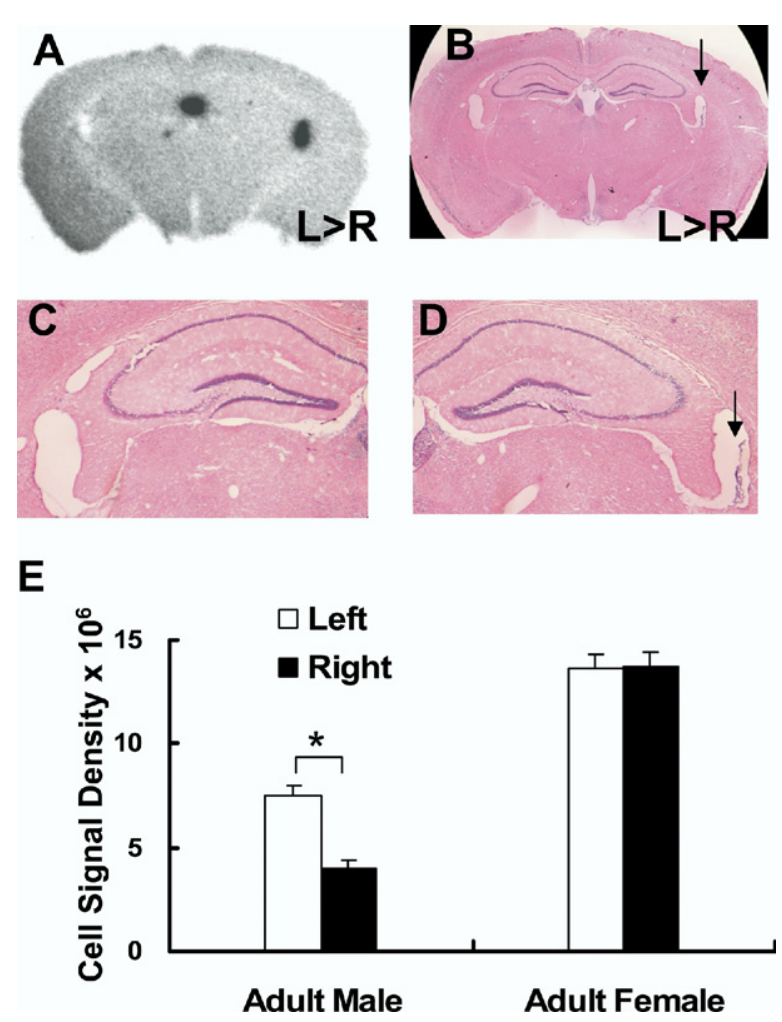

Fig. 3. Distribution of the choroid plexus cells in the brain of an adult male mouse. A section from the brain of an adult male mouse was analyzed by in situ hybridization to locate the TTR transcripts (A) and by $\mathrm{HE}$ staining to locate the choroid plexus cells in the lateral ventricles (B). Magnified pictures of sub-regions containing the right and left ventricles in (B) are shown in (C) and (D), respectively. The arrows point to the choroid plexus cells that are visible in the left ventricle but not in the right ventricle. (E) The cell signal density of adult male $(n=3)$ and female $(n=3)$ mice. Data are expressed as means \pm standard deviation (SE). ${ }^{*} P<0.05$ by the $t$-test.

major functions of TTR, as suggested previously by others (for refs, see Discussion), was to scavenge soluble $A \beta$ and thus help to prevent the amyloid plaque aggregation, it would be interesting to examine the expression patterns of TTR in the two hemispheres of aged mice as well as in a mouse model of $A D$.

The levels of TTR transcripts in the hemispheres of five each of aged C57BL/6JNarl (24-month-old) male and female mice were analyzed by real time RT-PCR. As seen in Fig. 4, the distribution of TTR transcripts in the two hemispheres was found to be symmetrical in aged mice of either gender. When the levels of the hemispheric TTR transcript levels of the adult (2-month) and aged mice were compared, it was obvious that the levels of the brain TTR transcripts of the adult females, aged females, and aged males were all higher than the adult males (Fig. 4). Furthermore, in contrast to the latter, the distribution patterns of TTR transcripts in the hemispheres of the former four categories of mice were all mainly symmetrical.

Tg2576 mice overexpress the human amyloid precursor protein (APP) protein with the "Swedish" mutation, and they develop AD-like features such as the memory deficits and $A \beta$ plaques in the brain (Hsiao et al., 1996; Ashe, 


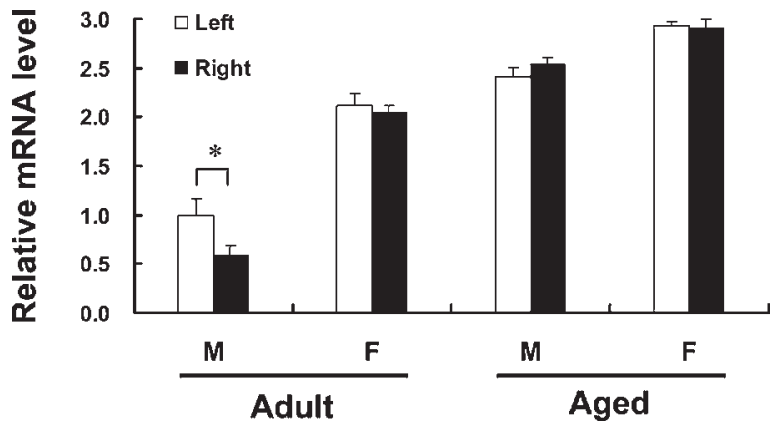

Fig. 4. Real time RT-PCR analysis of aged C57BL/6JNarl mice in comparison to 2-month adult mice. Total RNAs from the left and right cerebral hemispheres of 10 each of 24-month male mice, 24-month female mice, 2-month male mice, and 2-month female mice were analyzed by real time RT-PCR and compared. The expression levels were normalized to GAPDH. Note the leftward asymmetry of TTR transcript distribution was observed only in the adult males $\left({ }^{*} P<0.05\right.$ by the $t$-test). Data are expressed as means \pm SE.

2001, Westerman et al., 2002). Immediately after birth, the level of $A \beta$ in the $\operatorname{Tg} 2576$ mouse brain was already severalfold higher than that of the normal mice, and it increased rapidly at 6 months of age (Kawarabayashi et al., 2001; Apelt et al., 2004). The amyloid plaques began to accumulate in the brains of Tg2576 mice around the age of 9 months when the AD-like features started to develop (Kawarabayashi et al., 2001). Furthermore, the expression level of TTR was high in Tg2576, which was suggested to be the result of gene reprogramming due to the overexpression of APP and/or its metabolites such as $A \beta$ (Stein and Johnson, 2002). It has also been suggested that the relatively high level of TTR confers neuro-protection of Tg2576 before the age of 9-10 months (Stein and Johnson, 2002). These characteristics made Tg2576 suitable as one of the animal models for examining the relationship among TTR, A $\beta$, and the pathological development of AD. Since the Morris water maze learning task has indicated behavior deficiency of the 10-month-old Tg2576 mice, but not Tg2576 mice of the ages 2 and 6 months, respectively (Westerman et al., 2002; data not shown), we have analyzed by real time RT-PCR the expression levels of TTR in the cerebral hemispheres of 2-, 6-, and 10-month, as well as 24-month-old Tg2576 mice in comparison to their nontransgenic littermates (Fig. 5).

As shown, the levels of TTR transcripts in the brains of adult Tg2576 mice of the ages 2, 6 and 10 months, respectively, were all significantly higher than the non-transgenic mice (Fig. 5). Interestingly, the leftward-asymmetry of TTR transcript distribution observed in the corresponding age groups of the male non-transgenic mice (Fig. 5B) or wild-type adult males (Fig. 4) was absent in the adult males of Tg2576 (Fig. 5A). Remarkably, the 10-month-old male Tg2576 mice even exhibited a rightward-asymmetry pattern of TTR transcript distribution in their hemispheres (Fig. 5A). On the contrary, the expression levels of TTR transcripts in the brain of 24-month-old Tg2576 mice of either gender (Fig. 5A) were significantly less than the corresponding age groups of either the non-transgenic mice (Fig. 5B) or the wild-type ones (Fig. 4). These data suggested that not only the level, but also the hemisphericsymmetry of TTR expression in the mouse brain was significantly altered in the AD mouse model Tg2576.

\section{$A \beta$ levels in the brains of $\mathrm{Tg} 2576$ mice}

The TTR expression pattern in the brains of 10-month-old male Tg2576 mice, but not those of the age 2, 6 or 24 months, showed a rightward-asymmetry in the cerebral hemispheres. We have examined the $A \beta$ levels in the male and female Tg2576 mice of different ages. Interestingly, the levels of both the SDS-A $\beta$ and fa-A $\beta$ in the two hemispheres of these mice were found to be asymmetrical in the 10-month male Tg2576 mice, but they were symmetrical otherwise (Fig. 6). These data suggested that during disease progression of the male Tg2576 mice, the $A \beta$ in the left hemispheres of the 10-month-old male Tg2576 mice accumulated to a higher level than the right one and this likely led to the lower level of TTR in the left hemisphere of these mice, as shown already above Fig. 5 .

\section{DISCUSSION}

It is well known that the mammalian brains are asymmetrical structurally and functionally, and brain asymmetry is linked with several neurological diseases. As shown above, with a combined use of different approaches, we have identified TTR, a gene previously correlated with the prevention of the AD pathogenesis, as one of the genes asymmetrically expressed in the adult mouse brain.

It has been reported before that the level of human brain TTR increases in aged people due to the increases of
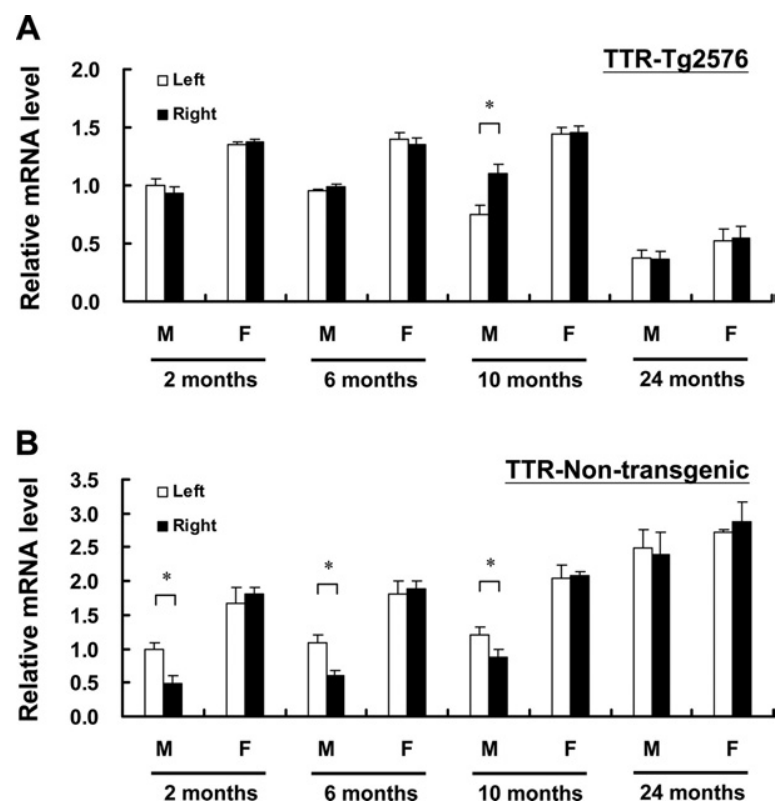

Fig. 5. Real time RT-PCR analysis of the cerebral hemispheres of Tg2576 and non-transgenic mice. The expression of the TTR transcripts in the hemispheres of $\operatorname{Tg} 2576$ (A) and non-transgenic (B) mice of different genders and different ages (five mice each) was analyzed by real time RT-PCR. Gene expression was normalized to GAPDH. The data are expressed as means \pm SE, ${ }^{*} P<0.05$ by the $t$-test. 

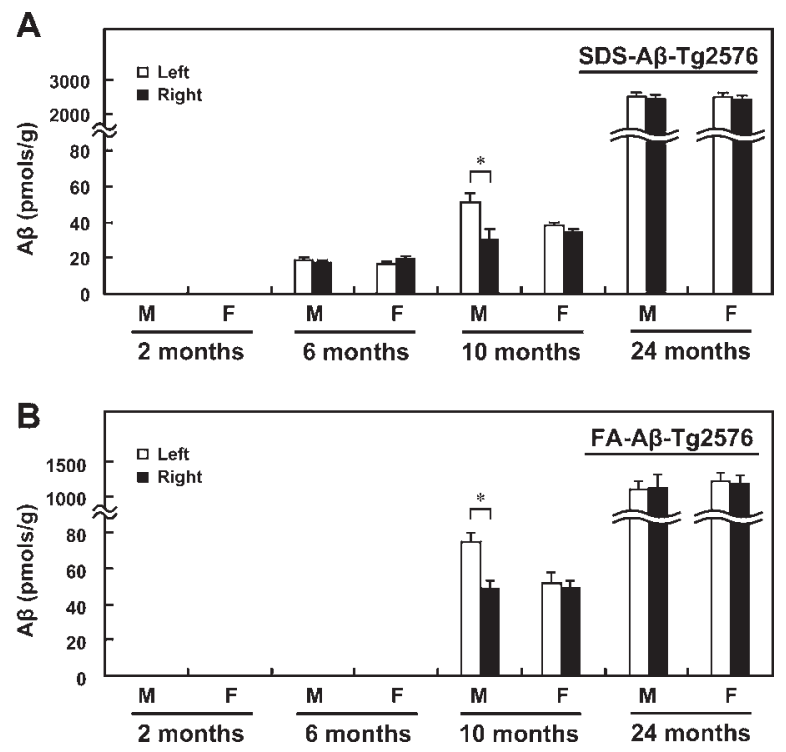

Fig. 6. $A \beta$ levels of the cerebral hemispheres in the 2-, 6-, 10-month and 24 -month-old Tg2576 mice. The SDS-A $\beta$ levels (A) and fa- $A \beta$ levels (B) in the hemispheres of Tg2576 mice of different genders (five mice each) were analyzed by ELISA test. Note that there were no detectable SDS-A $\beta$ in the 2-month-old Tg2576 mice, and no fa-A $\beta$ in the 2 and 6 -month-old ones. The data are expressed as means \pm SE, ${ }^{*} P<0.05$ by the $t$-test.

its secretion and filtration by the choroid plexus, passive diffusion through the BBB, and reabsorption by the BBB (Merched et al., 1998; Chodobski and SzmydyngerChodobska, 2001). In AD patients, as already mentioned in the introduction, the TTR level increases at the early stage of pathogenesis as the result of activation of the TTR gene expression by $A \beta$ (Stein and Johnson, 2002), but it decreases with the development of $A D$ due to killing of the choroid plexus cells by the vast amount of the $A \beta$ aggregates (Serot et al., 1997; Chodobski and SzmydyngerChodobska, 2001). Animal studies, on the other hand, have shown that the brains of female mice contain higher levels of TTR, likely due to estrogen activating TTR gene expression in the choroid plexus cells (Tang et al., 2004). In consonance with the above studies in literatures, we have found in our analysis a higher level of brain TTR expression in normal aged mice (24-month-old) than the adults (2 to 10-month-old) (Fig. 4 and Fig. 5B), in female mice than the males (Figs. 4 and 5), and in Tg2576 AD mice of the age 2-10 months than the non-transgenic controls (Fig. 5).

Notable in the adult mice, however, not only the level of TTR expression in the whole brain but also the hemispheric distribution of the TTR transcripts differed between the two genders (Figs. 1-3, Table 1). The strong lateralization of TTR expression in the male mouse brain, as evidenced by real time RT-PCR data of the hemispheric RNAs (Fig. 1), the in situ hybridization patterns of the ventricles (Fig. 2A, 2B), and Western blotting (Fig. 2C) is in interesting correlation with the more lateralized brain structure of the male mammals than the females (Dewing et al., 2003). The asymmetric distribution of the TTR transcripts in the ventricles resulted mainly from the asymmetric distribution of the epithelial cells in the left and right choroid plexus (Fig. 3). This asymmetric TTR expression in the adult mouse hemispheres, however, is not an all-or-none phenomenon in the population (Table 1). This is somewhat similar to several aspects of the known structural and cognitive asymmetries of the mammalian brain ( $\mathrm{Ge}$ schwind and Levitsky, 1968; Galaburda et al., 1978; Jürgens and Zwirner, 2000). Our finding of the asymmetry in the distributions of the epithelia cells in the choroid plexus and, by implication, the local organization of the left ventricles represents a newly identified brain asymmetry on the cellular level.

What might be the functional implications of the asymmetric distribution of the TTR transcripts in the male brains? As mentioned in the introduction, TTR could carry thyroid hormone $\mathrm{T}_{4}$ into the CNS. It also associates with retinol-binding protein for the transport of vitamin A. Thus, the asymmetric expression of TTR may indirectly affect the levels of free thyroid hormones and/or vitamin A uptake by the peripheral tissues, although there was no noted abnormality in the brains of TTR gene knockout mice except $T_{4}$ distribution (Episkopou et al., 1993; Palha et al., 1997).

More interestingly, the observed male-dominant, leftward asymmetry of TTR distribution in the brains of adult mice and, by inference, the human adults might have a functional link with AD pathogenesis. Since TTR could bind and sequester sA $\beta$ (Schwarzman et al., 1994; Link, 1995), the lowering of the levels of the CSF TTR in most, if not all, late-stage AD patients (Serot et al., 1997; Merched et al., 1998; Chodobski and Szmydynger-Chodobska, 2001) as well as in the 24-month Tg2576 mice (Fig. 5A) could contribute significantly to the disease progression. Furthermore, several characteristics of the AD brains are associated with hemispheric asymmetries, which include the temporal asymmetry in patients with delusion thoughts (Geroldi et al., 2000a; Sultzer et al., 2003), a marked leftward ventricular asymmetry in the AD patients as well as in the elderly controls (Toga and Thompson, 2003), and the faster rate of the local gray matter loss in the left hemispheres in AD patients (Thompson et al., 2003). In view of the above and the expression data of TTR in the brains of Tg2576 mice in comparison to the non-transgenic controls (Fig. 5), we suggest the following scenarios to account for the gender-, age-, and AD pathogenesis-dependent patterns of TTR gene expression in the two hemispheres of the mouse brain. First, in the normal adult females, the levels of TTR expression on both sides of the brain are higher than the adult males (Fig. 4 and Fig. 5B), which has been shown previously to be at least in part due to the stimulation by estrogen (Tang et al., 2004). This relatively high level of TTR could protect both hemispheres of the adult females from accumulation of the $A \beta$ aggregates. Secondly, in the normal adult males, the characteristics of a higher level of TTR in the left hemisphere of the rodents (Fig. 4 and Fig. 5B) and, by inference, human may have been evolutionarily selected to play a preventive role helping to reduce $A \beta$ plaque formation in the region. This is consistent with the observation that cellular damage in the 
human $A D$ brain is more severe in the left hemisphere (Thompson et al., 2003; Toga and Thompson, 2003). This higher susceptibility of the left brain to $A \beta$-induced neurodegeneration is reflected by the higher level of $A \beta$ in the left hemisphere of 10-month Tg2576 mice (Fig. 6). Interestingly, the higher level of $A \beta$ aggregate in the left brains of 10-month Tg2576 mice is accompanied by a rightward asymmetry of TTR expression (Fig. 5A), which is most likely due to the damage of epithelial cells of the choroid plexus in the left hemisphere by the $A \beta$ aggregates.

Finally, when the animals age, as in the normal (Fig. 4) and non-transgenic mice (Fig. $5 B$ ), or when the $A \beta$ aggregates accumulate, as in the adult Tg2576 (Fig. 5A), expression of the TTR gene is enhanced in both hemispheres of either gender. At the mean time, the leftward asymmetry of TTR transcript distribution in the adult male brains is also lost (Figs. 4 and 5). When the amount of the $\mathrm{A} \beta$ becomes too high, as in the 24-month Tg2576 mice (Kawarabayashi et al., 2001; Apelt et al., 2004), to be effectively sequestered by TTR, the toxicity of the aggregated $A \beta$ would cause significant deaths of the epithelial cells of the choroids plexus and the consequent decrease of TTR expression (Fig. 5A). Similar events could occur in female AD patients passing the menopause stage, when the synthesis of estrogen stops.

In summary, we have identified TTR, a gene previously suggested to play a role in the reduction of $A D$, as a leftward gene asymmetrically expressed in the brains of the adult male mice, and by implication in human adult males. In view of the known pathological features of human $A D$ and our data of TTR expression in the normal mice and an $A D$ mouse model, we have suggested possible scenarios for the observed patterns of TTR expression in the brains of the two genders and changes of these patterns during normal development as well as during neurodegeneration caused by excess $A \beta$. The absolute as well as relative levels of TTR in the two hemispheres might be a good indicator for pathogenesis of $A D$.

Acknowledgments-We thank Dr. Da-Fu Chen for the discussion with him in the National Taiwan University Hospital. This study was supported by the Academia Sinica, Taipei, Taiwan. K.-J. Tsai is an Academia Sinica Distinguished Postdoctoral Scholar, and C.-K.J. Shen is an Academia Sinica Investigator Awardee.

\section{REFERENCES}

Apelt J, Bigl M, Wunderlich P, Schliebs R (2004) Aging-related increase in oxidative stress correlates with developmental pattern of $\beta$-secretase activity and $\beta$-amyloid plaque formation in transgenic Tg2576 mice with Alzheimer-like pathology. Int J Dev Neurosci 22:475-484.

Ashe $\mathrm{KH}$ (2001) Learning and memory in transgenic mice modeling Alzheimer's disease. Learn Mem 8:301-308.

Barta PE, Pearlson GD, Brill LB II, Royall R, McGilchrist IK, Pulver AE, Powers RE, Casanova MF, Tien AY, Frangou S, Petty RG (1997) Planum temporale asymmetry reversal in schizophrenia: replication and relationship to gray matter abnormalities. Am J Psychiatry 154:661-667.

Buxbaum JN, Ye Z, Reixach N, Friske L, Levy C, Das P, Golde T, Masliah E, Roberts AR, Bartfai T (2008) Transthyretin protects
Alzheimer's mice from the behavioral and biochemical effects of A $\beta$ toxicity. Proc Natl Acad Sci U S A 105:2681-2686.

Chanoine JP, Alex S, Fang SL, Stone S, Leonard JL, Korhle J, Braverman LE (1992) Role of transthyretin in the transport of thyroxine from the blood to the choroid plexus, the cerebrospinal fluid, and the brain. Endocrinology 130:933-938.

Chodobski A, Szmydynger-Chodobska J (2001) Choroid plexus: target for polypeptides and site of their synthesis. Microsc Res Tech 52:65-82.

Dewing P, Shi T, Horvath S, Vilain E (2003) Sexually dimorphic gene expression in mouse brain precedes gonadal differentiation. Mol Brain Res 118:82-90.

Episkopou V, Maeda S, Nishiguchi S, Shimada K, Gaitanaris GA, Gottesman ME, Robertson EJ (1993) Disruption of the transthyretin gene results in mice with depressed levels of plasma retinol and thyroid hormone. Proc Natl Acad Sci U S A 90:2375-2379.

Galaburda AM, LeMay M, Kemper TL, Geschwind N (1978) Right-left asymmetries in the brain. Science 199:852-856.

Geroldi C, Akkawi NM, Galluzzi S, Ubezio M, Binetti G, Zanetti O, Trabucchi M, Frisoni GB (2000a) Temporal lobe asymmetry in patients with Alzheimer's disease with delusions. J Neurol Neurosurg Psychiatry 69:187-191.

Geroldi C, Laakso MP, DeCarli C, Beltramello A, Bianchetti A, Soininen $\mathrm{H}$, Trabucchi M, Frisoni GB (2000b) Apolipoprotein E genotype and hippocampal asymmetry in Alzheimer's disease: a volumetric MRI study. J Neurol Neurosurg Psychiatry 68:93-96.

Geschwind DH, Miller BL (2001) Molecular approaches to cerebral laterality: development and neurodegeneration. Am J Med Genet 101:370-381.

Geschwind N, Levitsky W (1968) Human brain: left-right asymmetries in temporal speech region. Science 161:186-187.

Hsiao K, Chapman P, Nilsen S, Eckman C, Harigaya Y, Younkin S, Yang F, Cole $G$ (1996) Correlative memory deficits, $A \beta$ elevation, and amyloid plaques in transgenic mice. Science 274:99-102.

Hugdahl K (2000) Lateralization of cognitive processes in the brain. Acta Psychol 105:211-235.

Jürgens U, Zwirner P (2000) Individual hemispheric asymmetry in vocal fold control of the squirrel monkey. Behav Brain Res 109:213-217.

Kawakami R, Shinohara $Y$, Kato $Y$, Sugiyama H, Shigemoto R, Ito I (2003) Asymmetrical allocation of NMDA receptor $\epsilon 2$ subunits in hippocampal circuitry. Science 300:990-994.

Kawarabayashi T, Younkin LH, Saido TC, Shoji M, Ashe KH, Younkin SG (2001) Age-dependent changes in brain, CSF, and plasma amyloid $\beta$ protein in the Tg2576 transgenic mouse model of Alzheimer's disease. J Neurosci 21:372-381.

Lein ES, Hawrylycz MJ, Ao N, Ayres M, Bensinger A, Bernard A, Boe AF, Boguski MS, Brockway KS, Byrnes EJ, et al. (2007) Genomewide atlas of gene expression in the adult mouse brain. Nature 445:168-176.

Link CD (1995) Expression of human $\beta$-amyloid peptide in transgenic Caenorhabditis elegans. Proc Natl Acad Sci U S A 92:9368-9372.

Lipshutz RJ, Fodor SP, Gingeras TR, Lockhart DJ (1999) High density synthetic oligonucleotide arrays. Nat Genet 21:20-24.

Merched A, Serot JM, Visvikis S, Aguillon D, Faure G, Siest G (1998) Apolipoprotein E, transthyretin and actin in the CSF of Alzheimer's patients: relation with the senile plaques and cytoskeleton biochemistry. FEBS Lett 425:225-228.

Palha JA, Episkopou V, Maeda S, Shimada K, Gottesman ME, Saraiva MJM (1994) Thyroid hormone metabolism in a transthyretin-null mouse strain. J Biol Chem 269:33135-33139.

Palha JA, Hays MT, de Morreale de Escobar G, Episkopou V, Gottesman ME, Saraiva MJ (1997) Transthyretin is not essential for thyroxine to reach the brain and other tissues in transthyretin-null mice. Am J Physiol 272:E485-E493.

Pantel J, Kratz B, Essig M, Schröder J (2003) Parahippocampal volume deficits in subjects with aging-associated cognitive decline. Am J Psychiatry 160:379-382. 
Schreiber G (2002) The evolutionary and integrative roles of transthyretin in thyroid hormone homeostasis. J Endocrinol 175:61-73.

Schwarzman AL, Gregori L, Vitek MP, Lyubski S, Strittmatter WJ, Enghilde JJ, Bhasin R, Silverman J, Weisgraber KH, Coyle PK, Zagorski MG, Talafous J, Eisenberg M, Saunders AM, Roses AD, Goldgaber D (1994) Transthyretin sequesters amyloid beta protein and prevents amyloid formation. Proc Natl Acad Sci U S A 91:8368-8372.

Serot JM, Christmann D, Dubost T, Couturier M (1997) Cerebrospinal fluid transthyretin: aging and late onset Alzheimer's disease. J Neurol Neurosurg Psychiatry 63:506-508.

Sousa JC, Cardoso I, Marques F, Saraiva MJ, Palha JA (2007) Transthyretin and Alzheimer's disease: where in the brain? Neurobiol Aging 28:713-718.

Stauder AJ, Dickson PW, Aldred AR, Schreiber G, Mendelsohn FAO, Hudson P (1986) Synthesis of transthyretin (pre-albumin) mRNA in choroid plexus epithelial cells, localized by in situ hybridization in rat brain. J Histochem Cytochem 34:949-952.

Stein TD, Johnson JA (2002) Lack of neurodegeneration in transgenic mice overexpression mutant amyloid precursor protein is associated with increased levels of transthyretin and the activation of cell survival pathways. J Neurosci 22:7380-7388.

Stein TD, Anders NJ, DeCarli C, Chan SL, Mattson MP, Johnson JA (2004) Neutralization of transthyretin reverses the neuroprotective effects of secreted amyloid precursor protein (APP) in APP sw $_{\text {mice }}$ resulting in tau phosphorylation and loss of hippocampal neurons: support for the amyloid hypothesis. J Neurosci 24:7707-7717.

Sultzer DL, Brown CV, Mandelkern MA, Mahler ME, Chen ST, Cummings JL (2003) Delusional thought and regional frontal/temporal cortex metabolism in Alzheimer's disease. Am J Psychiatry 160:341-349.

Sun T, Patoine C, Abu-Khalil A, Visvader J, Sum E, Cherry TJ, Orkin SH, Geschwind DH, Walsh CA (2005) Early asymmetry of gene transcription in embryonic human left and right cerebral cortex. Science 308:1794-1798.

Sun T, Walsh CA (2006) Molecular approaches to brain asymmetry and handedness. Nat Rev Neurosci 7:655-662.

Tabibnia G, Cooke BM, Breedlove SM (1999) Sex difference and laterality in the volume of mouse dentate gyrus granule cell layer. Brain Res 827:41-45.

Tang YP, Haslam SZ, Conrad SE, Sisk CL (2004) Estrogen increases brain expression of the mRNA encoding transthyretin, an amyloid beta scavenger protein. J Alzheimers Dis 6:413-420.

Thompson PM, Hayashi KM, de Zubicaray G, Janke AL, Rose SE, Semple J, Herman D, Hong MS, Dittmer SS, Doddrell DM, Toga AW (2003) Dynamics of gray matter loss in Alzheimer's disease. J Neurosci 23:994-1005.

Toga AW, Thompson PW (2003) Mapping brain asymmetry. Nat Rev Neurosci 4:37-48.

Tsai KJ, Chen SK, Ma YL, Hsu WL, Lee EH (2002) sgk, A primary glucocorticoid-induced gene, facilitates memory consolidation of spatial learning in rats. Proc Natl Acad Sci U S A 99:3990-3995.

Tsai KJ, Tsai YC, Shen CK (2007) G-CSF rescues the memory impairment of animal models of Alzheimer's disease. J Exp Med 204:1273-1280.

Westerman MA, Cooper-Blacketer D, Mariash A, Kotilinek L, Kawarabayashi T, Younkin LH, Carlson GA, Younkin SG, Ashe KH (2002) The relationship between $A \beta$ and memory in the Tg2576 mouse model of Alzheimer's disease. J Neurosci 22:1858-1867. 